\title{
Conditions for a consistent picture of critical phenomena in a strongly coupled ionic mixture
}

\author{
D. Léger (*) and C. Deutsch \\ Laboratoire de Physique des Gaz et des Plasmas (**), \\ Université Paris XI, Bâtiment 212, 91405 Orsay Cedex, France
}

(Reçu le ler février 1985, accepté le 15 avril 1985)

\begin{abstract}
Résumé. - Nous analysons les conditions minimales requises pour obtenir une description cohérente du phénomène de démixtion dans un mélange ionique binaire. Nous avons mis en évidence le rôle fondamental joué par la polarisation électronique. Nous montrons que si la fonction diélectrique électronique satisfait à la règle de somme sur la compressibilité et si l'hypothèse du couplage faible électron-ion est remplie, alors le mélange ionique peut être décrit comme un mélange de "pseudo-atomes".
\end{abstract}

\begin{abstract}
We discuss the minimum conditions required to build a consistent picture for the demixing phenomena in a binary ionic mixture. We show that the electronic polarization plays a fundamental role. Namely, if the dielectric electronic function $\varepsilon(q)$ fulfils the compressibility sum rule and if the weak-coupling electron-ion hypothesis applies, then the ionic mixture may be described as a mixture of « pseudo-atoms".
\end{abstract}

\section{Introduction.}

In the statistical mechanics of strongly coupled ionic mixtures, it remains to put on a sound basis the conditions required to observe demixing. Up to now this important problem has been investigated within the framework of the Binary Ionic Mixture (BIM) model [1-3] where the neutralizing electron fluid is taken as non-responding and mechanically rigid. Our claim is that this background should be allowed through polarization to follow the enhanced ionic fluctuations near criticality in order to obtain a coherent microscopic picture near the demixing transition.

Previous attempts such as that of Baus [14] have shown demixing within the BIM model only by hypothesizing an ad hoc behaviour for the ion-ion structure factors. Recently Totsuji et al. [5] have introduced polarizability in the electron fluid and conveniently extended the scope of the one-component plasma (OCP) model to weakly coupled $\left(r_{\mathrm{s}} \leqslant 1\right)$ Jellium, well below any critical phenomenon.

In this work we consider strongly coupled ionic mixtures at any $r_{\mathrm{s}}$ value compatible with the weak coupling electron ion (WCH) hypothesis. We analyse the polarized BIM (PBIM) within the

(*) Also Conservatoire National des Arts et Métiers, 292, rue St-Martin, 75141 Paris Cedex 03, France.

$(* *)$ Associé au C.N.R.S. 
framework of the theory of thermodynamic fluctuations (TTF). We show that the PBIM model exhibits the main characteristics of a neutral mixture in the long wavelength limit $(k \rightarrow 0)$ provided the electronic dielectric function fulfils the compressibility sum rule.

\section{Description of the PBIM model.}

We are concerned with a plasma consisting of two ionic species of charge number $Z_{\alpha}(\alpha=1$ refers to the smallest charge) and a neutralizing electron gas. The ionic component is parametrized as follows : we denote by $\rho_{\mathrm{i}}$ the total ionic number density $\left(\rho_{\mathrm{i}}=\lim _{V \rightarrow \infty} N_{\mathrm{i}} / V\right)$ and by $c_{\alpha}$ the concentration number of the $\alpha$ species. We choose the ion-sphere radius $a_{\mathrm{i}}=\left(3 / 4 \pi \rho_{\mathrm{i}}\right)^{1 / 3}$ as the unit of length and we define the plasma parameter as $\Gamma=\beta e^{2} / a_{\mathrm{i}}$ with $\beta=\left(k_{\mathrm{B}} T\right)^{-1}$.

The electron gas is characterized by the usual dimensionless parameter $r_{\mathrm{s}}=a_{\mathrm{e}} / a_{0}$ where $a_{0}$ is the Bohr radius, $a_{\mathrm{e}}=a_{\mathrm{i}} /\langle Z\rangle^{1 / 3}$ and $\left\langle Z^{v}\right\rangle=\sum_{\alpha=1}^{2} c_{\alpha} Z_{\alpha}^{v}$ defines an average charge number.

In the PBIM the linear response of the electron gas is introduced through the static dielectric function $\varepsilon(k)$. For our present purpose we define a quantity of central interest which we denote by $\Delta \hat{v}(k)$ and write as :

$$
\Delta \hat{v}(k)=\hat{v}(k)-\left.\beta \rho_{\mathrm{i}} \frac{\partial \mu_{\mathrm{e}}}{\partial \rho_{\mathrm{e}}}\right|_{T} \equiv \hat{v}(k)-\frac{1}{\langle Z\rangle^{2}} \frac{K_{0}}{K_{\mathrm{e}}}
$$

where $\hat{v}(k)=\frac{4 \pi \beta \rho_{\mathrm{i}} e^{2}}{\varepsilon(k) k^{2}}$ is the screened ion-ion potential, $K_{\mathrm{e}}$ denotes the electronic compressibility and $K_{0}=\beta / \rho_{\mathrm{i}}$.

It is easily verified that if $\varepsilon(k)$ fulfils the electronic compressibility sum rule [6] then $\Delta \hat{v}(k)$ exhibits the following long wavelength limit :

$$
\lim _{k \rightarrow 0} \Delta \hat{v}(k)=0\left(\sim-k^{2}\right)(\text { PBIM }) \text {. }
$$

In the opposite case the BIM is governed by the singular long wavelength behaviour of the Coulomb potential while $\varepsilon(k)=1$ as a consequence of the non-responding character of the electron gas. Therefore $\Delta \hat{v}(k)$ behaves like $k^{-2}$ and is singular in the limit $k \rightarrow 0$.

According to the WCH we approximate the thermodynamic variables of the whole system as the sum of the electronic and ionic components. Thus with evident notations, we get, $p=p_{\mathrm{e}}+p_{\mathrm{i}}$, $K^{-1}=K_{\mathrm{e}}^{-1}+K_{\mathrm{i}}^{-1}, g=g_{\mathrm{e}}+g_{\mathrm{i}}$ for the pressure, the inverse compressibility and the Gibbs free energy respectively. Due to the electroneutrality we write $g$ as

$$
g=\sum_{\alpha=1}^{2} c_{\alpha} \mu_{\mathrm{i} \alpha}+\langle Z\rangle \mu_{\mathrm{e}}=\sum_{\alpha=1}^{2} c_{\alpha} \tilde{\mu}_{\alpha}
$$

defining the effective chemical potentials $\tilde{\mu}_{\alpha}$ of the whole system.

As will be detailed elsewhere, the afore-mentioned superposition rule allows us to describe the PBIM in two equivalent forms as we work with the ionic or with the entire system variables. In the following, we choose for convenience the latter formulation.

\section{The TTF applied to the PBIM model.}

Hereafter we extend the TTF analysis of Vieillefosse et al. [7] to the case of a polarizable ionic mixture. 
In a fully isolated PBIM of fixed volume and temperature, the total entropy variation which arises from any local macroscopic density fluctuation $\delta \rho_{\mathrm{i} \alpha}(\mathbf{r})$ reads :

$$
\Delta S / k_{\mathrm{B}}=-\left(E_{\mathrm{c}}+E_{\mathbf{p}}\right)-\frac{\beta}{2 V} \sum_{\alpha=1}^{2} \sum_{\mathbf{k}}^{\prime} \delta \hat{\mu}_{\mathrm{i} \alpha}(\mathbf{k}) \delta \hat{\rho}_{\mathrm{i} \alpha}(\mathbf{k})
$$

with :

$$
\begin{aligned}
& E_{\mathrm{c}}=\frac{1}{2 V} \sum_{\alpha \beta=1}^{2} \sum_{\mathbf{k}}^{\prime} Z_{\alpha} Z_{\beta} \frac{4 \pi \beta e^{2}}{k^{2}} \delta \hat{\rho}_{\mathrm{i} \alpha}(\mathbf{k}) \delta \hat{\rho}_{\mathrm{i} \beta}(\mathbf{k}) \\
& E_{\mathrm{P}}=\frac{1}{2 V} \sum_{\alpha \beta=1}^{2} \sum_{\mathbf{k}}^{\prime} Z_{\alpha} Z_{\beta} \frac{4 \pi \beta e^{2}}{k^{2}}\left(\frac{1}{\varepsilon(k)}-1\right) \delta \hat{\rho}_{\mathrm{i} \alpha}(\mathbf{k}) \delta \hat{\rho}_{\mathrm{i} \beta}(\mathbf{k})
\end{aligned}
$$

where we denote by $\delta \hat{f}(\mathbf{k})$ the Fourier transform of $\delta f(\mathbf{r})$.

The physical meaning of equations (4) and (5) is that under the density fluctuations which we consider, the internal energy of the system is transformed into a Coulombic macroscopic energy $E_{\mathrm{c}}$ and a term $E_{\mathrm{p}}$ which we add to account for the polarization (or band structure) energy of the electron gas taken in the linear response approximation [8].

We thus express $\Delta S$ as a fundamental quadratic form in the density fluctuations :

$$
\Delta S / k_{\mathrm{B}}=-\frac{1}{2} \sum_{\alpha \beta=1}^{2} \sum_{\mathbf{k}}^{\prime}\left(c_{\alpha} c_{\beta}\right)^{1 / 2}\left(Z_{\alpha} Z_{\beta} \Delta \hat{v}(\mathbf{k})+\beta \rho_{\mathrm{i}} \tilde{\mu}_{\alpha \beta}\right) \frac{\delta \hat{\rho}_{\mathrm{i} \alpha}(\mathbf{k}) \delta \hat{\rho}_{\mathrm{i} \beta}(\mathbf{k})}{\left(N_{\mathrm{i} \alpha} N_{\mathrm{i} \beta}\right)^{1 / 2}}
$$

where the short notation $\tilde{\mu}_{\alpha \beta}$ stands for the derivative $\left.\frac{\partial \tilde{\mu}_{\alpha}}{\partial \rho_{\mathrm{i} \beta}}\right|_{T}, N_{\mathrm{i} \alpha}=c_{\alpha} N_{\mathrm{i}}$ and $\Delta \hat{v}(k)$ is the effective potential which we have already defined in the previous section (Eq. (1)).

Equation (6) plays a major role in the derivation of the long wavelength limit of the ionic structure factors as well as to analyse the stability conditions of the whole system [9].

3. 1 Stability CONDitions. - For the system to be stable against the density fluctuations the expression (6) must be a definite negative quadratic form. This implies the two following inequalities :

$$
\begin{aligned}
& \text { 1. } \frac{K_{0}}{K}+\langle Z\rangle^{2} \Delta \hat{v}(k) \geqslant 0 \quad k \ll a_{\mathrm{i}}^{-1} \\
& \text { 2. }\left.\frac{K_{0}}{K} \beta \frac{\partial^{2} g}{\partial c_{1}^{2}}\right|_{T_{\mathrm{p}}}+\langle Z\rangle^{2} \Delta \hat{v}(k)\left(\left.\beta \frac{\partial^{2} g}{\partial c_{1}^{2}}\right|_{T_{\mathrm{p}}}+\frac{K_{0}}{K} \tilde{\delta}^{\prime 2}\right) \geqslant 0 \quad k \ll a_{\mathrm{i}}^{-1} .
\end{aligned}
$$

Here $\widetilde{\delta}^{\prime}=\left.\frac{1}{\rho_{\mathrm{e}}} \frac{\partial \rho_{\mathrm{e}}}{\partial c_{1}}\right|_{T_{\mathrm{p}}}$ stands for an electronic expansion coefficient.

The conditions (7a) and (7b) are readily derived starting from equation (6) which is first reexpressed in the couple of variables $\left(\delta \hat{\rho}_{\mathrm{i}}(k), \delta \hat{c}_{1}(k)\right)$ while a few thermodynamic relationships are to be used.

As usual, we refer to (7a) and (7b) as the mechanical and interdiffusion stability condition (ISC), respectively. It is an easy matter to verify that the above stability criteria applied in the PBIM are exactly the same as in a neutral mixture. Namely the total compressibility is a definite positive quantity while the ISC reads as usual as $\left.\beta \frac{\partial^{2} g}{\partial c_{1}^{2}}\right|_{T_{\mathbf{p}}} \geqslant 0$. 
In the opposite case, the BIM exhibits a peculiar behaviour due to the singular long wavelength limit of the Coulomb potential. The ionic compressibility has no definite sign, as is well-known, and nothing is said about the total compressibility. Moreover equation (7b) indicates that the ISC now reads as :

$$
\left.\beta \frac{\partial^{2} g}{\partial c_{1}^{2}}\right|_{T_{\mathrm{p}}}+\tilde{\delta}^{\prime 2} \frac{K_{0}}{K} \geqslant 0 .
$$

One can state that the term $\tilde{\delta}^{\prime 2} \frac{K_{0}}{K}$ remains finite even in the limit $r_{\mathrm{s}} \rightarrow 0$. This point gives us some insight into the atypical behaviour of the BIM versus the critical phenomenon and leads us to expect the PBIM to be more efficient, at least in the " microscopic approach » to demixing.

3.2 THE IONIC STRUCTURE FACTORS IN THE THERMODYNAMIC LIMIT. - The long wavelength limit of the structure factors $S_{\alpha \beta}$ are easily derived starting from expression (6) for the entropy. Denoting by $H_{\alpha \beta}$ the inverse matrix of the $S_{\alpha \beta}$, s, we get :

$$
H_{\alpha \beta}(k)=\left(c_{\alpha} c_{\beta}\right)^{1 / 2}\left(Z_{\alpha} Z_{\beta} \Delta \hat{v}(k)+\beta \rho_{\mathrm{i}} \tilde{\mu}_{\alpha \beta}\right) \quad k \ll a_{\mathrm{i}}^{-1}
$$

and therefore :

$$
S_{\alpha \beta}(k)=\frac{\varepsilon_{\alpha \beta}}{D(k)}\left(c_{\bar{\alpha}} c_{\bar{\beta}}\right)^{1 / 2}\left(Z_{\bar{\alpha}} Z_{\bar{\beta}} \Delta \hat{v}(k)+\beta \rho_{\mathrm{i}} \tilde{\mu}_{\bar{\beta} \bar{\alpha}}\right) \quad k \ll a_{\mathrm{i}}^{-1}
$$

with $\bar{\alpha}=3-\alpha$ and $\varepsilon_{\alpha \beta}=1$ if $\alpha=\beta,-1$ if not.

The denominator $D(k)$ in equation (10) is equal to the determinant of the matrix $H_{\alpha \beta}$ :

$$
D(k)=c_{1} c_{2}\left[\left.\beta \frac{\partial^{2} g}{\partial c_{1}^{2}}\right|_{T_{\mathrm{p}}} \frac{K_{0}}{K}+\langle Z\rangle^{2} \Delta \hat{v}(k)\left(\frac{K_{0}}{K}{\delta^{\prime 2}}^{2}+\left.\beta \frac{\partial^{2} g}{\partial c_{1}^{2}}\right|_{T_{\mathrm{p}}}\right)\right]
$$

which we have already encountered previously (Eq. (7b)).

The one ionic component version of equation (10) reduces to :

$$
S(k)=\left[Z^{2} \Delta \hat{v}(k)+\left.\beta \rho_{\mathrm{i}} \frac{\partial \tilde{\mu}}{\partial \rho_{\mathrm{i}}}\right|_{T}\right]^{-1} \equiv\left[Z^{2} \hat{v}(k)+\frac{K_{0}}{K_{\mathrm{i}}}\right]^{-1} .
$$

It has been recently obtained by Totsuji et al. [5] with another method and successfully compared with the Monte Carlo « experiment » results they derived in a polarized OCP.

As a consequence of equations (10) and (11), the critical behaviour of the $S_{\alpha \beta}$ 's is driven by the actual properties of $D(k)$. It is easily verified that in the PBIM all the structure factors behave like $\left(\left.\beta \frac{\partial^{2} g}{\partial c_{1}^{2}}\right|_{T_{\mathrm{p}}}\right)^{-1}$. This result holds for the concentration-concentration structure factor

$$
S_{\mathrm{cc}}(k)=c_{1} c_{2}\left(\sum_{\alpha \beta=1}^{2} \varepsilon_{\alpha \beta}\left(c_{\alpha} c_{\beta}\right)^{1 / 2} S_{\overline{\alpha \beta}}(k)\right)
$$

and we get :

$$
\lim _{k \rightarrow 0} S_{\mathrm{cc}}(k)=\left[\left.\beta \frac{\partial^{2} g}{\partial c_{1}^{2}}\right|_{T_{\mathrm{p}}}\right]^{-1}
$$

exactly as in a neutral mixture [10]. It holds as well for the charge-charge structure factor 
$S_{Z Z}(k)=\left(\sum_{\alpha \beta=1}^{2}\left(c_{\alpha} c_{\beta}\right)^{1 / 2} Z_{\alpha} Z_{\beta} S_{\alpha \beta}(k)\right) /\left\langle Z^{2}\right\rangle$ which is now finite in the limit $k \rightarrow 0$, a result which agrees with the sum rule :

$$
\lim _{k \rightarrow 0} S_{z Z}(k) / \varepsilon(k)=0
$$

which will be detailed elsewhere.

In the opposite case, the BIM exhibits anew a distinct feature : the left hand side of the inequality (8) replaces $\left.\beta \frac{\partial^{2} g}{\partial c_{1}^{2}}\right|_{T_{\mathrm{p}}}$ in the denominator of $S_{\mathrm{cc}}$ and the $S_{\alpha \beta}$ 's while $S_{z z}$ vanishes as $k^{2}$, as a consequence of electroneutrality.

The afore-mentioned analogy between the PBIM and a neutral-like mixture holds equally well for the compressibility relationship :

$$
\frac{K_{0}}{K}=1-\lim _{k \rightarrow 0} \sum_{\alpha \beta=1}^{2} c_{\alpha} c_{\beta} \hat{C}_{\alpha \beta}(k) \quad \text { (PBIM) }
$$

while the direct correlation function $\hat{C}_{\alpha \beta}(k)$ are deduced from the $H_{\alpha \beta}$ 's [4] :

$$
\hat{C}_{\alpha \beta}(k)=\left(\delta_{\alpha \beta}-H_{\alpha \beta}(k)\right) /\left(c_{\alpha} c_{\beta}\right)^{1 / 2} .
$$

It must be noted however that this analogy is lost when one expands $D(k)$ in power of $k^{2}$. Denoting by $\xi$ a correlation length, we find $D(k)$ to behave like $\left(k^{2}-\xi^{-2}\right)$ while $\Delta \hat{v}(k)$ vanishes as $-k^{2}$ (see e.g. Eq. (2)). Therefore the pair correlation functions $h_{\alpha \beta}(r)$ exhibit an undamped oscillatory tail like $\cos (\xi r) / r$ in the limit $r \rightarrow \infty$. This remains a peculiar feature of the PBIM.

\section{Numerical results.}

In the present work the electronic polarization effects have been studied within the framework of the $\mathrm{HNC}$ theory applied to a fully ionized $\mathrm{H}^{+}-\mathrm{H}_{\mathrm{e}}^{2+}$ mixture. The $\mathrm{HNC}$ closure equations complemented with the Ornstein-Zernike relation were solved iteratively in order to get the ionic structure factors both in the BIM and in the PBIM model. In the latter case we have retained the dielectric functions of Thomas-Fermi, Lindhard and Hubbard [11] to account for electron screening.

We are mainly concerned with the microscopic demixing approach through the properties of the denominateur $D(k)$ (Eq. (11)). In order to compare the BIM versus the PBIM critical behaviour, we find it better to compute the normalized quantity $D_{0}=\lim _{k \rightarrow 0} D(k) /\left\langle Z^{2}\right\rangle \hat{v}(k)$. We choose the set $\left(\Gamma, r_{\mathrm{s}}, c_{2}\right)$ as independent variables.

The HNC results for the BIM are displayed in table I. $c_{2}$ ranges from 0 to $100 \% H_{\mathrm{e}}$ and $\Gamma$ ranges from 0 to 150. As expected, $D_{0}$ has no significant deviation from unity, a result which agrees quite well with the particular character of the BIM which we have previously mentioned. This is to be contrasted with the PBIM behaviour pictured in figure 1 , As $r_{\mathrm{s}}$ increases, $D_{0}$ is lowered from the $\operatorname{BIM}\left(r_{\mathrm{s}}=0\right)$ value down to zero, which characterizes a critical phenomenon. In the Hubbard case (Fig. 1) we get the following critical values : $r_{\mathrm{s}} \approx 1.43, c_{2} \approx 0.75$ when $\Gamma=60$.

The following comments are in order :

$\alpha_{1}$. We have checked that the compressibility $K$ (Eq. (15)) remains positive when $r_{\mathrm{s}}$ ranges from zero to its critical value. Moreover $K^{-1}$ vanishes simultaneously with $D_{0}$ for the case of pure phases which also indicates a critical opalescent phenomenon to occur in this case.

$\alpha_{2}$. The HNC results are very sensitive to the choice of the dielectric function while the $r_{\mathrm{s}}$ critical value is more readily reached in the Hubbard case. We expect likewise the so-called brigde 
Table I. - HNC results for $D_{0}$ in the $\mathrm{H}^{+}-\mathrm{He}^{2+}$ binary ionic mixture $\left(r_{\mathrm{s}}=0\right)$.

\begin{tabular}{|c|c|c|c|c|c|c|c|c|c|}
\hline$c_{2}=\% H_{\mathrm{e}}$ & 0.000 & 0.125 & 0.250 & 0.375 & 0.500 & 0.625 & 0.750 & 0.875 & 1.000 \\
\hline$\Gamma=1$ & 1.000 & 0.983 & 0.980 & 0.981 & 0.984 & 0.988 & 0.992 & 0.996 & 1.000 \\
20 & 1.000 & 0.961 & 0.950 & 0.953 & 0.959 & 0.968 & 0.979 & 0.989 & 1.000 \\
40 & 1.000 & 0.957 & 0.946 & 0.947 & 0.955 & 0.964 & 0.975 & 0.988 & 1.000 \\
60 & 1.000 & 0.955 & 0.943 & 0.944 & 0.951 & 0.961 & 0.973 & 0.986 & 1.000 \\
80 & 1.000 & 0.954 & 0.941 & 0.941 & 0.948 & 0.958 & 0.970 & 0.984 & 1.000 \\
100 & 1.000 & 0.953 & 0.940 & 0.939 & 0.946 & 0.955 & 0.968 & 0.982 & 1.000 \\
150 & 1.000 & 0.950 & 0.937 & 0.936 & 0.942 & 0.951 & 0.963 & 0.979 & 1.000 \\
\hline
\end{tabular}

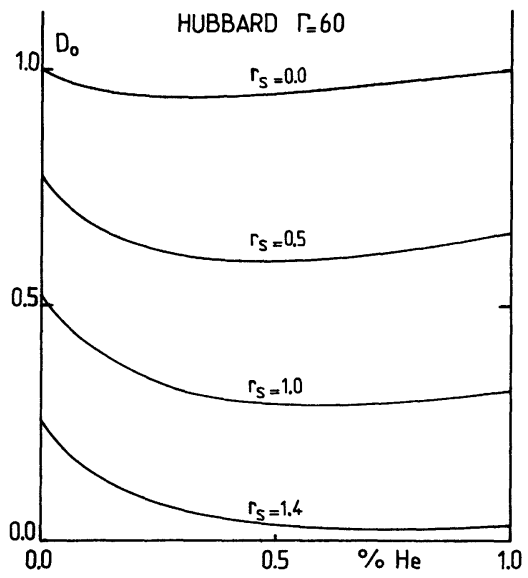

Fig. 1. - $\mathrm{HNC}$ results for $D_{0}$ in the $\mathrm{H}^{+}-\mathrm{He}^{2+}$ polarized binary ionic mixture with the Hubbard dielectric function.

functions, neglected in the HNC theory, to have a marked effect in the determination of the critical parameters.

\section{Conclusion.}

We have shown that the electronic polarization plays a fundamental role when studying the critical phenomena occurring in the ionic mixtures. Our previous numerical results agree quite well with the prediction of the TTF which has permitted us to link the PBIM to a pseudo-atom like mixture.

\section{Acknowledgments.}

We wish to thank D. Levesque and J. J. Weis for useful discussions. 


\section{References}

[1] Hansen, J. P., Torrie, G. M. and Vieillefosse, P., Phys. Rev. A 16 (1977) 2153.

[2] Pollock, E. L. and Alder, B. J., Phys. Rev. A 15 (1977) 1263.

[3] Stevenson, D. J., Phys. Rev. B 12 (1975) 3999.

[4] Baus, M., Phys. Rev. A 16 (1977) 2493.

[5] Totsui, H. and Tokami, K., Phys. Rev. A 30 (1984) 3175.

[6] PInes, D. and Nozières, P., The theory of quantum liquids (Benjamin, New York), Vol. I (1966).

[7] Vieillefosse, P. and Hansen, J. P., Phys. Rev. A 12 (1975) 1106. See e.g. Vieillefosse, P., Thèse de Doctorat d'Etat, Université Pierre et Marie Curie, Paris (1979).

[8] Ashcroft, N. W., Third. Int. Conf. on liquid metals (1976). Inst. Phys. Conf. Ser. No. 30 (1977) 39, edited by R. Evans and D. A. Greenwood.

[9] Landau, L. D. and Lifshitz, E. M., Statistical Physics (Pergamon, Oxford) 1959.

[10] Bhatia, A. B. and Thornton, D. E., Phys. Rev. B 2 (1970) 3004.

[11] Hubbard, J., Proc. R. Soc. A 243 (1957) 336. 